\title{
Screening for frailty in primary care: a systematic review of the psychometric properties of the frailty index in community-dwelling older people
}

Irene Drubbel ${ }^{1 *}$, Mattijs E Numans ${ }^{1,2}$, Guido Kranenburg ${ }^{1}$, Nienke Bleijenberg ${ }^{1}$, Niek J de Wit ${ }^{1}$ and Marieke J Schuurmans ${ }^{3}$

\begin{abstract}
Background: To better accommodate for the complex care needs of frail, older people, general practitioners must be capable of easily identifying frailty in daily clinical practice, for example, by using the frailty index (FI). To explore whether the $\mathrm{Fl}$ is a valid and adequate screening instrument for primary care, we conducted a systematic review of its psychometric properties.

Methods: We searched the Cochrane, PubMed and Embase databases and included original studies focusing on the criterion validity, construct validity and responsiveness of the FI when applied in community-dwelling older people. We evaluated the quality of the studies included using the Quality in Prognosis Studies (QUIPS) tool. This systematic review was conducted based on the PRISMA statement.

Results: Of the twenty studies identified, eighteen reported on Fls derived from research data, one reported upon an Fl derived from an administrative database of home-care clients, and one reported upon an Fl derived from routine primary care data. In general, the Fl showed good criterion and construct validity but lacked studies on responsiveness. When compared with studies that used data gathered for research purposes, there are indications that the FI mean score and range might be different in datasets using routine primary care data; however, this finding needs further investigation.
\end{abstract}

Conclusions: Our results suggest that the Fl is a valid frailty screening instrument. However, further research using routine Electronic Medical Record data is necessary to investigate whether the psychometric properties of the Fl are generalizable to a primary care setting and to facilitate its interpretation and implementation in daily clinical practice.

Trial registration: PROSPERO systematic review register number: CRD42013003737.

Keywords: Frailty, Systematic review, Psychometric properties, Primary care, Screening, Older patients

\section{Background}

Among other issues, ageing within the population poses a major burden on healthcare due to the increasing prevalence of frailty among older people [1]. Frailty is defined as a state of increased vulnerability due to somatic, environmental or psychosocial factors [2]. To better accommodate for the complex care needs of frail, older

\footnotetext{
* Correspondence: i.drubbel@umcutrecht.nl

'Department of General Practice, Julius Center for Health Sciences and Primary Care, University Medical Center Utrecht, Str. 6.131, Universiteitsweg 100, 3584 CG Utrecht, the Netherlands

Full list of author information is available at the end of the article
}

people, a transition towards proactive, population-based care is required, which will improve clinical outcomes and cost-effectiveness $[3,4]$. To facilitate this care transition, general practitioners (GPs) must be capable of identifying frail older patients within their daily clinical practice.

The Frailty Index (FI) is one of the screening tools for frailty [5]. An FI comprises a list of health deficits (e.g. symptoms, signs, impairments, and diseases) that are indicative of frailty. The proportion of deficits present forms the patient's FI score, which can range from zero to one [6]. When an FI consists of at least 30 deficits,

\section{Biomed Central}


different numbers and types of deficits may be used without major influence on the properties of the FI, which enables application in and comparison between different datasets [7].

There is considerable debate over whether the FI can be used for frailty screening in daily primary care. Some authors have stated that the FI has not been validated in this setting, that the instrument is of limited value due to its perceived complexity, that the FI has only moderate discriminative ability, and that other frailty instruments, such as the Tilburg Frailty Indicator, are more promising [8-11]. Others have argued that the FI is a significant predictor of adverse health outcomes, that it covers all important frailty factors, that it can be easily derived from routine administrative healthcare data, and they have called for further exploration of the FI's merits in primary care [12-14].

To further assess the potential of the FI as a screening and monitoring instrument for frailty in primary care, knowledge of its characteristics is essential. Therefore, we performed a systematic review of the literature and assessed the psychometric properties of the FI in identifying frailty among community-dwelling older people.

\section{Methods}

\section{Search strategy, selection criteria and data extraction}

We searched the Cochrane, PubMed, and Embase databases using the terms 'frailty AND (index OR deficit OR deficits OR cumulative OR accumulation)'. We searched for studies published from August $8^{\text {th }}, 2001$ onwards, which is the publication date of the landmark study presenting the FI concept [6]. The search was limited to studies in English, and databases were searched until October $30^{\text {th }}, 2012$. The first and third author (ID and GK) screened titles and abstracts independently and selected studies for full-text assessment. These full-text studies were assessed by the first author for inclusion, and in cases where doubt existed, an independent assessment by the last author (MS) followed. Citations from the included articles were also searched for additional relevant publications by the first author. Eligibility disagreements were resolved by consensus.

Studies were included that met the following criteria: first, the studies focused on an FI. The FI was defined as a list of health deficits for which patients were screened and that provided an FI score that reflected the proportion of deficits present on the predefined list [6]; second, only original research was included that assessed one of the following psychometric properties of the FI: criterion validity, construct validity or responsiveness; third, the studies focused primarily on community-dwelling older people. Community-dwelling older people were defined as older people who lived independently at home; older people who lived at home while receiving home care; and older people living in assisted living facilities. In the Netherlands, GPs provide care to older people in all these different living situations, and virtually all older people in these living situations are inscribed in a general practice. Studies were excluded when the FI was based on a comprehensive geriatric assessment (CGA), because it is not feasible to perform a CGA for all older patients in general practice. Also, studies were excluded when the entire study population was living in a nursing home, was hospitalized or was selected because of one specific disease in common. Secondary reports of FI datasets that did not report additional psychometric properties were excluded (see Additional file 1 for full details of inclusion and exclusion criteria). Based on these predefined criteria, the first author extracted data on general study characteristics, frailty index characteristics and assessed psychometric properties.

\section{Psychometric properties- definitions}

Currently, there is no consensus about a frailty reference standard against which the criterion validity of the FI could be assessed. However, since there is general agreement that the concept of frailty reflects a state of increased vulnerability to adverse health outcomes, criterion validity is defined as the ability of an FI to predict adverse health outcomes [15]. An Area Under the Curve (AUC) of $<0.70$ was considered poor; an AUC of 0.70-0.89 was considered adequate; and an AUC of $\geq 0.90$ was considered excellent [16]. Construct validity refers to the coherence of the FI with other frailty measures or related conditions and constructs, including comorbidity, disability, self-rated health, age, and gender [15]. Responsiveness reflects the ability of the FI to detect clinically important changes over time in the frailty construct (see Additional file 1 for a detailed description of the various psychometric properties) [17].

\section{Quality assessment}

Study quality was evaluated using the Quality in Prognosis Studies (QUIPS) tool, which considers six potential domains of bias: inclusion, attrition, prognostic factor measurement, confounders, outcome measurement, and analysis and reporting [18]. Each domain comprises a number of prompting items, which enable assessment of the domain as having a high, moderate or low risk of bias.

The QUIPS tool was considered the most appropriate quality appraisal tool because, conceptually, the frailty index is a prognostic instrument. We modified three domains of the QUIPS tool. First, in our review, we were interested only in the descriptive, rather than explanatory, relationships of the FI to adverse health outcomes and other measures; thus, we considered the domain 'confounders' irrelevant. Second, the domain 'outcome measurement' only accommodated studies in which the FI correlated with adverse outcomes, i.e., criterion 
validity studies. We modified this domain such that the QUIPS tool also applied to studies in which the FI was correlated cross-sectionally or longitudinally with other frailty measures or related constructs, i.e., construct validity or responsiveness studies. Third, in the domain 'prognostic factor measurement', we redefined the prompting item 'Valid and Reliable Measurement of Prognostic Factor' as 'Valid and Reliable Construction of Prognostic Factor' because the FI deficit list must be constructed based on specific criteria [2,19]: first, deficits should be acquired and related to health status; thus, 'blue eyes' is not an appropriate deficit whereas 'heart failure' is appropriate; second, deficit prevalence should increase with age; third, deficits should not 'saturate' too early, for example, presbyopia is present in almost all older people, thus, it is not appropriate as a deficit; fourth, the combination of deficits in an FI should cover a range of systems; fifth, the same FI should be used in follow-up measures; and finally, the FI should comprise at least 30 deficits and deficit prevalence should be at least 1\% [2] (see Additional file 2 for the modified QUIPS form that was used for the quality appraisal of the studies included).

\section{Registration}

This systematic review was registered prospectively in the PROSPERO international prospective register of systematic reviews (CRD42013003737).

\section{Funding}

This research was supported by a grant from ZON-MW, The Netherlands Organization for Health Research and Development (reference 311040201). The sponsor had no influence on the research design, data collection, data interpretation, the writing of this report or the decision to publish.

\section{Results}

Search results

After removing duplicates, our search resulted in 867 studies (Figure 1). We excluded 809 studies after screening the titles/abstracts and 38 studies after full-text assessment. We have listed the full bibliographic details and the reason for exclusion of each of these studies (available upon request). No additional studies were found in manual reference searching; thus, we used twenty studies for our final review.

\section{Description of study characteristics}

One study was a cross-sectional study [20], and nineteen studies were cohort studies with a follow-up ranging from one to twelve years (Table 1). Eighteen studies used survey datasets; one study used an administrative dataset of home-care clients [21], and one study was based on the analysis of routine administrative primary care data [22].

In ten studies, the study population was populationbased, consisting of a representative mixture of independently living and institutionalized older people, with the majority of people living independently [6,23-31]. Eight studies included only independently living older people [19,20,22,32-36]; and two studies focused specifically on older people receiving home care or older people in assisted living facilities [21,37]. The number of participants ranged from 754 to 36,424 older people with a mean age varying from 70.1 to 84.9 years, and the percentage of women varied from 50.0 to $76.7 \%$.

The FIs used in the studies were based on 13 to 92 health deficits. Most studies scored deficits dichotomously [6,21-26,29-31]. Eight studies applied multilevel scoring [19,28,32-37] and used, for example, a Likertscale [33]. Two studies did not report how the deficits were scored $[20,27]$. Two studies assigned extra weight to predefined deficits $[23,31]$, for example, to 'polypharmacy' [31]. The mean FI scores varied from 0.13 to 0.26 , and except for two studies that reported a lower maximum FI score [22,31], the maximum reported FI score varied from 0.60 to 0.70 .

\section{Quality assessment}

Four studies showed a low risk of bias for each of the five domains of the QUIPS tool considered, namely inclusion, attrition, prognostic factor measurement, outcome measurement, and analysis and reporting. Fourteen studies showed a moderate-to-high risk of bias in one or two domains; and two studies showed a moderate-to-high risk of bias in three or four domains (Table 2). Risks of bias were highest in the domain of study attrition, which was due to very low response rates [31] or an unclear response rate $[19,25,34]$. In one cohort study, attrition was not assessed because only the cross-sectional study component was considered [27]. For the remaining fourteen cohort studies, losses to follow-up were $<16 \%$.

In the domain of prognostic factor measurement, eleven studies were judged as having a moderate risk of bias $[19,20,22,24,27,28,30-32,34,36]$. Of these eleven studies, four studies did not report their entire FI deficit list $[20,26,27,32]$, three used data-driven cut-off points for the FI $[24,26,30]$, and nine did not report the percentage of missing FI data or how missing FI data were managed $[19,20,22,24,30-32,34,36]$. In the remaining nine studies showing a low risk of bias in the prognostic factor measurement, eight reported a percentage of missing data of $<5 \%[21,23,25,28,29,33,35,37]$, and one study did not report the percentage of missing data [6]. Six studies managed missing data by excluding the missing deficits from the denominator when calculating the FI $[6,25,28,32,35,37]$. Two studies imputed the missing FI data $[23,29]$. All 


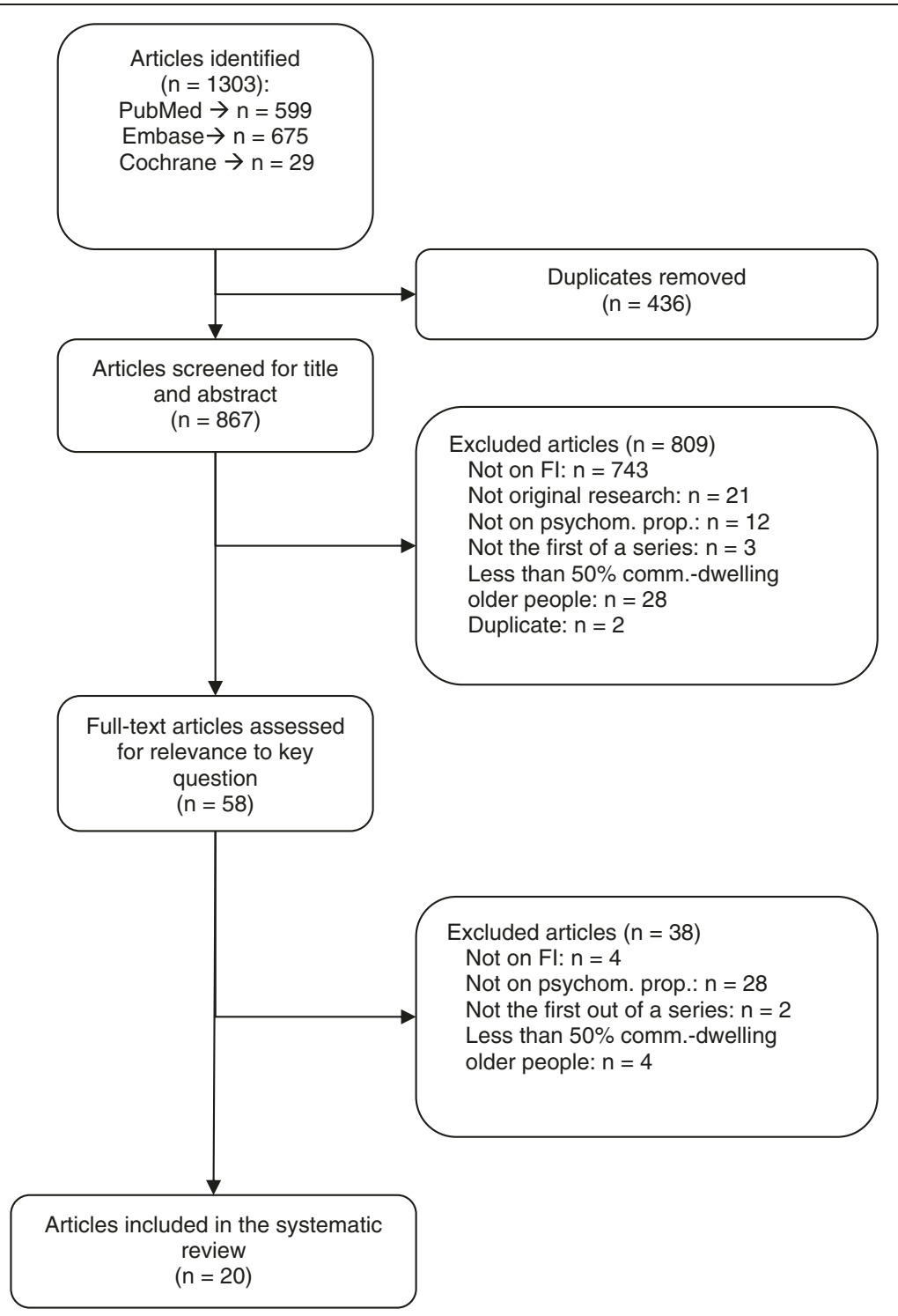

Figure 1 Flowchart of search results.

twenty studies complied with the criteria for adequate FI construction as described in the 'Methods' section.

In total, in the 20 included studies, $5.1 \%$ of domains, i.e., inclusion, attrition, prognostic factor measurement, outcome measurement, and analysis and reporting as assessed with the QUIPS tool showed a high risk of bias, $25.5 \%$ of domains showed a moderate risk of bias, and $69.4 \%$ of domains showed a low risk of bias (full QUIPS appraisal forms for each study are available upon request).

\section{Psychometric properties of the FI Criterion validity}

Fifteen studies assessed the criterion validity of the FI by evaluating the predictive ability of the FI for mortality, institutionalization, hospitalization, number of days in hospital, morbidity, Emergency Department (ED) visits, out-of-hours GP consultations, falls, fractures, change in ADL score, and change in mental score (Table 3). In each study, the FI was incorporated into a multivariable regression model that was corrected for age, gender and a variety of other co-variables. In each model, the FI was a significant predictor of the assessed outcome.

Twelve studies focused on the prediction of mortality, for which hazard ratios of 1.01 ( $\mathrm{SE} \pm 0.003$; per deficit increase in the frailty index) to 6.45 (95\% CI 4.10-10.14, most-frail group (FI score $0.35-0.65$ ) versus the leastfrail group (FI score $<0.07)$ were reported [34,33]. A multivariable model with age, gender, co-morbidity and an FI resulted in an Area Under the Curve (AUC) of 
Table 1 General characteristics of the studies included

\begin{tabular}{|c|c|c|c|c|c|c|c|c|}
\hline \multirow[t]{2}{*}{ Study } & \multirow[t]{2}{*}{ Design data set } & \multirow{2}{*}{$\begin{array}{l}\text { Total } N \text { (\% women) } \\
\text { Mean age (yrs } \pm S D) \\
\text { Setting }\end{array}$} & \multirow{2}{*}{$\begin{array}{l}\text { Follow-up } \\
\text { (LTFU) }\end{array}$} & \multicolumn{3}{|c|}{ FI deficits } & \multicolumn{2}{|c|}{ FI scores } \\
\hline & & & & $\begin{array}{l}\text { Deficit } \\
\text { number }\end{array}$ & $\begin{array}{l}\text { Deficit } \\
\text { scoring }\end{array}$ & $\begin{array}{c}\text { Deficit } \\
\text { weighting }\end{array}$ & $\begin{array}{c}\text { Mean/Median } \\
( \pm \mathrm{SD} / \mathrm{IQR})\end{array}$ & Range \\
\hline \multirow{3}{*}{$\begin{array}{l}\text { Armstrong } \\
\text { et al. [21] }\end{array}$} & \multirow{2}{*}{$\begin{array}{l}\text { Retrospective cohort } \\
\text { study }\end{array}$} & $23,952(69.4 \%)$ & $1 \mathrm{yr}(?)$ & 50 & B & No & $?$ & $?-0.66$ \\
\hline & & $81.7( \pm 7.4)$ & & & & & & \\
\hline & 8 CCACs & Home-care clients & & & & & & \\
\hline \multirow{3}{*}{$\begin{array}{l}\text { Cigolle et al. } \\
\text { [20] }\end{array}$} & Cross-sectional study & $1,657(55.5 \%)$ & N/A & 38 & $?$ & $?$ & $?$ & $?$ \\
\hline & & $?$ & & & & & & \\
\hline & HRS & Community-dwelling & & & & & & \\
\hline \multirow{3}{*}{$\begin{array}{l}\text { Drubbel et al. } \\
\text { [22] }\end{array}$} & \multirow{2}{*}{$\begin{array}{l}\text { Retrospective cohort } \\
\text { study }\end{array}$} & $1,679(59 \%)$ & 2 yrs (10.5\%) & 36 & B & No & $0.08(0.03-0.14)$ & $0-0.42$ \\
\hline & & Median 73 (IQR 65-81) & & & & & & \\
\hline & GPs EMRs & Community-dwelling & & & & & & \\
\hline \multirow{3}{*}{$\begin{array}{l}\text { Fang et al. } \\
{[32]}\end{array}$} & \multirow{2}{*}{$\begin{array}{l}\text { Retrospective cohort } \\
\text { study }\end{array}$} & $3,257(51.1 \%)$ & 8 yrs (13.8\%) & 33 & $\mathrm{~B} / \mathrm{M}$ & No & $0.13( \pm ?)$ & $0-0.67$ \\
\hline & & $70.1( \pm 9.0)$ & & & & & & \\
\hline & BLSA & Community-dwelling & & & & & & \\
\hline \multirow{5}{*}{$\begin{array}{l}\text { García- } \\
\text { González } \\
\text { et al. [33] }\end{array}$} & Retrospective & Total sample: 4,872 & 1.95 yrs (13.2\%) & 34 & $\mathrm{~B} / \mathrm{M}$ & No & $0.16( \pm 0.11)$ & $0-0.65$ \\
\hline & cohort study & Analyzed sample: & & & & & & \\
\hline & MHAS & $4,082(52.5 \%)$ & & & & & & \\
\hline & & 73 (range 65-105) & & & & & & \\
\hline & & Community-dwelling & & & & & & \\
\hline \multirow[t]{3}{*}{ Gu et al. [23] } & \multirow{2}{*}{$\begin{array}{l}\text { Retrospective cohort } \\
\text { study }\end{array}$} & $13,861(57.2 \%)$ & 3 yrs (12.9\%) & 39 & B & Yes & $0.26( \pm ?)$ & $?$ \\
\hline & & ? (range 65-109) & & & & & & \\
\hline & CLHLS & Population-based & & & & & & \\
\hline \multirow{3}{*}{$\begin{array}{l}\text { Hogan et al. } \\
\text { [37] }\end{array}$} & \multirow{2}{*}{$\begin{array}{l}\text { Retrospective cohort } \\
\text { study }\end{array}$} & 1,066 (76.7\%) & 1 yr (0\%) & $83^{\mathrm{a}}$ & $\mathrm{B} / \mathrm{M}$ & No & $?$ & $?$ \\
\hline & & $84.9( \pm 7.3)$ & & & & & & \\
\hline & ACCES & Assisted living residents & & & & & & \\
\hline \multirow{3}{*}{$\begin{array}{l}\text { Kulminski } \\
\text { et al. [24] }\end{array}$} & \multirow{2}{*}{$\begin{array}{l}\text { Retrospective cohort } \\
\text { study }\end{array}$} & 4,721 (?\%) & 4 yrs (0\%) & 48 & $\mathrm{~B}$ & No & $?$ & $0-0.70$ \\
\hline & & $?$ & & & & & & \\
\hline & $\mathrm{CHS}$ & Population-based & & & & & & \\
\hline \multirow{3}{*}{$\begin{array}{l}\text { Kulminski } \\
\text { et al. [25] }\end{array}$} & \multirow{2}{*}{$\begin{array}{l}\text { Retrospective cohort } \\
\text { study }\end{array}$} & $24,206(65.9 \%)$ & 4 yrs (?) & 32 & $B$ & No & $0.25( \pm ?)$ & $0-0.70$ \\
\hline & & $78.3( \pm ?)$ & & & & & & \\
\hline & NLTCS & Population-based & & & & & & \\
\hline \multirow{3}{*}{$\begin{array}{l}\text { Lucicesare } \\
\text { et al. [27] }\end{array}$} & Prospective cohort study & $1,016(55.4 \%)$ & 4 yrs (0\%) & 43 & B & No & $0.14( \pm ?)$ & $0-0.70$ \\
\hline & & $74.7( \pm 7.1)$ & & & & & & \\
\hline & CSBA & Population -based & & & & & & \\
\hline Lucicesare & Retrospective cohort & $1,318(63.1 \%)$ & 5 yrs (?) & 38 & $?$ & $?$ & $?$ & $0-0.59$ \\
\hline et al. [28] & study & $76.05( \pm ?)$ & & & & & & \\
\hline & $\mathrm{CSHA}$ & Population-based & & & & & & \\
\hline Mitnitski & Retrospective cohort & $36,424(58.5 \%)$ & 3-12 yrs (?) & 10 Fl's: $38-40$ & $\mathrm{~B} / \mathrm{M}$ & No & $?$ & $?$ \\
\hline & $\begin{array}{l}\text { study NPHS, (SHA (3), } \\
\text { ALSA, SOPSA, NHANES, }\end{array}$ & 74 (range: 27 - 105) & & & & & & \\
\hline & $\begin{array}{l}\text { H-70, NLTCS-I, } \\
\text { ICONS, BCS }\end{array}$ & $\begin{array}{l}7 \text { community-dwelling } \\
\text { and } 4 \text { clinical/ } \\
\text { institutional samples }\end{array}$ & & $1 \mathrm{FI}: 13$ & & & & \\
\hline Mitnitski & Retrospective cohort & 2,913 (?\%) & 5 yrs (?) & 92 & B & No & $?$ & $?$ \\
\hline & & $82( \pm 7.4)$ & & & & & & \\
\hline & $\mathrm{CSHA}$ & Population-based & & & & & & \\
\hline
\end{tabular}


Table 1 General characteristics of the studies included (Continued)

\begin{tabular}{|c|c|c|c|c|c|c|c|c|}
\hline \multirow{3}{*}{$\begin{array}{l}\text { Rockwood } \\
\text { et al. [28] }\end{array}$} & \multirow{2}{*}{$\begin{array}{l}\text { Retrospective cohort } \\
\text { study }\end{array}$} & 2,305 (?\%) & 5 yrs (?) & \multirow[t]{3}{*}{70} & \multirow[t]{3}{*}{$\mathrm{B} / \mathrm{M}$} & \multirow[t]{3}{*}{ No } & \multirow[t]{3}{*}{$?$} & \multirow[t]{3}{*}{$0-0.70$} \\
\hline & & ? & $?$ & & & & & \\
\hline & $\mathrm{CSHA}$ & Population-based & & & & & & \\
\hline \multirow{3}{*}{$\begin{array}{l}\text { Searle et al. } \\
\text { [9] }\end{array}$} & \multirow{2}{*}{$\begin{array}{l}\text { Retrospective cohort } \\
\text { study }\end{array}$} & $754(64.6 \%)$ & 9 yrs (<10\%) & \multirow[t]{3}{*}{40} & \multirow[t]{3}{*}{$\mathrm{B} / \mathrm{M}$} & \multirow[t]{3}{*}{ No } & \multirow[t]{3}{*}{$?$} & \multirow[t]{3}{*}{$0-0.60$} \\
\hline & & $?$ & & & & & & \\
\hline & YPEP & Community-dwelling & & & & & & \\
\hline \multirow[t]{3}{*}{ Shi et al. [35] } & \multirow{2}{*}{$\begin{array}{l}\text { Retrospective cohort } \\
\text { study }\end{array}$} & $3,257((51.1 \%)$ & 8 yrs (12.2\%) & \multirow[t]{3}{*}{35} & \multirow[t]{3}{*}{$\mathrm{B} / \mathrm{M}$} & \multirow[t]{3}{*}{ No } & \multirow[t]{3}{*}{$?$} & \multirow[t]{3}{*}{$?-0.70$} \\
\hline & & $70.1( \pm 9.0)$ & & & & & & \\
\hline & BLSA & Community-dwelling & & & & & & \\
\hline \multirow{3}{*}{$\begin{array}{l}\text { Song et al. } \\
\text { [29] }\end{array}$} & \multirow{2}{*}{$\begin{array}{l}\text { Retrospective cohort } \\
\text { study }\end{array}$} & $2,740(60.8 \%)$ & 10 yrs (10.1\%) & \multirow[t]{3}{*}{36} & \multirow[t]{3}{*}{ B } & \multirow[t]{3}{*}{ No } & \multirow[t]{3}{*}{$0.15( \pm ?)$} & \multirow[t]{3}{*}{$0-0.70$} \\
\hline & & $74( \pm 6.6)$ & & & & & & \\
\hline & NPHS & Population-based & & & & & & \\
\hline \multirow{3}{*}{$\begin{array}{l}\text { Theou et al. } \\
\text { [36] }\end{array}$} & \multirow{2}{*}{$\begin{array}{l}\text { Retrospective cohort } \\
\text { study }\end{array}$} & 2,305 (62.1\%) & 5 yrs (?) & $\mathrm{FI} 1: 37^{\mathrm{b}}$ & $\mathrm{B} / \mathrm{M}$ & No & FI 2: $0.24( \pm 0.15)$ & $0-0.68$ \\
\hline & & $84.6( \pm 7.0)$ & & \multirow[t]{2}{*}{$\mathrm{FI} 2: 37^{c}$} & & & & \\
\hline & $\mathrm{CSHA}$ & Community-dwelling & & & & & & \\
\hline \multirow{3}{*}{$\begin{array}{l}\text { Woo et al. } \\
\text { [30] }\end{array}$} & \multirow{2}{*}{$\begin{array}{l}\text { Prospective cohort } \\
\text { study }\end{array}$} & $4,000(50 \%)$ & 4 yrs (15.9\%) & \multirow[t]{3}{*}{47} & $\mathrm{~B}$ & No & $?$ & $?$ \\
\hline & & $?$ & & & & & & \\
\hline & CUHKS & Community-dwelling & & & & & & \\
\hline Woo et al. & Retrospective cohort & 2,032 (50.8\%) & 10 yrs & 62 & B & Yes & $0.13(?)$ & $0-0.53$ \\
\hline & study & $?$ & $42.4 \%$ (3 yrs) & & & & & \\
\hline & HKHS & Population-based & 85.3\% (10 yrs) & & & & & \\
\hline
\end{tabular}

$?=$ no information found/unclear; ${ }^{\text {an }}$ this study, two Fls were assessed: the Armstrong index and the Full Frailty Index. Only the second FI is reported here (both FIs show similar results); ${ }^{b}$ Excluding ADLs/comorbidities, ${ }^{C}$ Including ADLs/comorbidities comprising 37 different deficits to $\mathrm{FI} 1 ; \mathrm{B}=$ binary scoring; FI = Frailty Index; $\mathrm{IQR}=$ Interquartile range; $\mathrm{LTFU}=$ Lost to follow-up; $\mathrm{M}=$ multilevel scoring; $\mathrm{N} / \mathrm{A}=$ not applicable; Population-based = representative sample of community-dwelling and institutionalized older people; SD = standard deviation; Data sources: ACCES = Alberta Continuing Care Epidemiological Studies; ALSA = Australian Longitudinal Study of Ageing; BCS = Breast Cancer Survivor Study; BLSA = Beijing Longitudinal Study of Ageing; CCAC = Community Care Access Centre;

$\mathrm{CHS}=$ Cardiovasculair Health Study; CLHLS = Chinese Longitudinal Healthy Longevity Survey; CSBA = Conselice Study of Brain Ageing; CSHA: Canadian Study of Health and Ageing; CUHKS = Chinese University of Hong Kong Study; GPs EMR = General Practitioners' Electronic Medical Record; $\mathrm{H}-70=\mathrm{Gothenburg}$ Study; HKHS = Hong Kong Health Survey; HKSPH = Hong Kong School of Public Health study; HRS = Health and Retirement Survey; ICONS = Improving Cardiovascular Outcomes in Nova Scotia; MHAS = Mexican Health and Aging Study; NHANES = National Health and Nutrition Examination Survey; NLTCS $(-\mathrm{i})=$ National Long Term Care Survey (-institute); NPHS = National Population Health Survey; SOPSA = Sydney Older Persons Studies on Aging; YPEP = Yale Precipitating Events Project.

0.691 (95\% CI 0.648-0.733) for one-year mortality [37]. Used as a single independent variable, the FI predicted two-year mortality with an AUC of $0.780( \pm 0.020 \mathrm{SE})$ and a ten-year mortality with an AUC of $0.720( \pm 0.020$ SE) [29].

For other outcome measures, comparable AUCs were as follows: 0.610 (95\% CI 0.576-0.644) for one-year hospitalization risk and 0.667 (95\% CI $0.625-0.707)$ for a one-year risk of moving to long-term care [37]. For the prediction of time to the combined outcome of ED/outof-hours GP surgery visits, nursing home admission and mortality, the c-statistic of the FI used as a single independent variable was 0.686 (95\% CI 0.664-0.708). When the FI was combined in a model with age, gender, and consultation gap, the c-statistic improved to 0.702 (95\% CI 0.680-0.724) [22].

One study tested the added value of the FI in a multivariable model for predicting adverse health outcomes. For mortality and transition to long-term care, the AUCs of the models including an FI were significantly higher than the AUCs of a model comprising only age, gender and co-morbidity $(\mathrm{p}<0.03)$. For hospitalization, the AUC of the full model with age, gender, co-morbidity and an FI was significantly higher than the AUC of a model comprising only age and gender $(p<0.001)$ [37].

\section{Construct validity}

Eleven studies evaluated the construct validity of the FI $[6,20,21,24-28,34,36,37]$. The FI showed a strong positive correlation with the Functional Reach test $(r=0.73)$ [29], Consolice Study of Brain Ageing (CSBA) score $(r=0.72)$ [26], Frailty Phenotype (0.65) [28], and Edmonton Frail Scale (EFS; $r=0.61$ ) [21], a strong negative correlation with the Mini Mental State Examination score $(r=-0.58)$ [28], and a moderate correlation with the Changes in Health, End-Stage Disease and Signs and Symptoms (CHESS) Scale $(r=0.35)$ [21]. When the dichotomized FI was compared with the Frailty Phenotype where the latter 
Table 2 Assessment of risk of bias using the 'Quality Assessment in Prognostic Studies' (QUIPS) tool

\begin{tabular}{|c|c|c|c|c|c|}
\hline Study & Study participation & Study attrition & Prognostic factor measurement & Outcome measurement & Statistical analysis \\
\hline Armstrong et al. [21] & Low & Low & Low & Moderate & Low \\
\hline Cigolle et al. [20] & Low & N/A & Moderate & Low & Moderate \\
\hline Drubbel et al. [22] & Low & Moderate & Moderate & Low & Low \\
\hline Fang et al. [32] & Low & Moderate & Moderate & Low & Low \\
\hline García-González et al. [33] & Low & Moderate & Low & Low & Low \\
\hline Gu et al. [23] & Low & Low & Low & Low & Low \\
\hline Hogan et al. [37] & Low & Low & Low & Low & Low \\
\hline Kulminski et al. [24] & Moderate & Low & Moderate & Low & Low \\
\hline Kulminski et al. [25] & Low & High & Low & Low & Low \\
\hline Lucicesare et al. [26] & Low & Low & Moderate & Low & Moderate \\
\hline Lucicesare et al. [27] & Low & $N / A^{a}$ & Moderate & Low & Low \\
\hline Mitnitski et al. [34] & Low & High & Moderate & Low & Low \\
\hline Mitnitski et al. [6] & Low & Moderate & Low & Low & Low \\
\hline Rockwood et al. [28] & Moderate & Moderate & Low & Low & Low \\
\hline Searle et al. [19] & Low & High & Moderate & Low & Low \\
\hline Shi et al. [35] & Low & Low & Low & Low & Low \\
\hline Song et al. [29] & Low & Low & Low & Low & Low \\
\hline Theou et al. [36] & Low & Moderate & Moderate & Low & Moderate \\
\hline Woo et al. [30] & High & Moderate & Moderate & Low & Moderate \\
\hline Woo et al. [31] & Low & High & Moderate & Low & Low \\
\hline
\end{tabular}

Low $=$ low risk of bias, Moderate $=$ moderate risk of bias, High $=$ high risk of bias. Level of risk of bias was determined by judgement of the prompting items belonging to each assessed domain. ${ }^{a}$ Attrition was not assessed because only the cross-sectional component in which construct validity was examined was of interest.

was used as a reference standard, the FI showed a sensitivity of 45.9 to $60.7 \%$ and a specificity of 83.5 to $90.0 \%$ $[20,24]$. When compared with the Functional Domains model, the sensitivity of the FI was $38 \%$, and its specificity was $91.5 \%$ [20]. When using a three-level risk categorization, the weighted kappa of the FI compared with the Frailty Phenotype was 0.17 (95\% CI 0.13-0.20), and the weighted kappa of the FI compared with the CHESS scale was 0.36 (95\% CI 0.31-0.40).

The FI displayed moderate correlation with the concept of self-rated health $(r=0.49)$, which was expressed as an index of self-rated health deficits [27]. When the crude correlation of the FI was assessed with age, a weak to moderate correlation of $0.193,0.241$ and 0.320 , respectively, was reported $[6,25,26]$. One study compared the age trajectories of the FI score within community-dwelling and institutional/clinical cohorts [34], with higher levels of comorbidity and disability in the latter. The FI score increased gradually with age in community-dwelling cohorts, whereas the FI score was high at all ages in the institutional/clinical cohorts.

One study examined specifically an FI with only symptoms and signs as deficits and demonstrated that older people with higher FI scores showed more functional impairments in (I) ADL and more co-morbidity than patients with lower FI scores [36].

Without formally assessing correlations within a construct validity context, sixteen studies reported that older people and women show higher FI scores $[6,19,20,22,23,25-37]$, and only one study reported a lower percentage of women in the most-frail group [21].

Six studies quantified the increase in FI score with chronological age, all reporting a similar increase in FI score with age ranging from +0.02 to $0.05 /$ year $[6,19,22,26,34,35]$.

No studies reported on the responsiveness of the FI in daily clinical practice.

\section{Discussion}

In this systematic review, we demonstrate that the FI adequately predicts a wide range of adverse health outcomes and that its discriminative capability is poor to adequate. The FI correlates strongly with other frailty measures, except for the CHESS scale. However, this scale is not a frailty measure per se but was designed to measure 'health instability' and to specifically predict mortality in institutionalized older people [38]. The FI score increases steadily with age, and the maximum FI 
Table 3 Criterion validity results; the predictive ability of the frailty index for adverse health outcomes

\begin{tabular}{|c|c|c|c|c|c|c|}
\hline Study & $\begin{array}{l}\text { Outcome variable with } \\
\text { events }(n)\end{array}$ & Model & Factors controlled for in model & Effect measure & $95 \% \mathrm{CI} / \mathrm{SE}$ & $\begin{array}{l}\text { Interpretation } \\
\text { effect measure }\end{array}$ \\
\hline \multirow[t]{3}{*}{ Armstrong et al. [21] } & Mortality: 1676 & \multirow{3}{*}{$\begin{array}{l}\text { Cox proportional } \\
\text { hazards regression }\end{array}$} & \multirow[t]{3}{*}{ Age, gender } & $\mathrm{Fl}: \mathrm{HR}=1.93$ & $1.79-2.08$ & \multirow{3}{*}{$\begin{array}{l}\text { Most frail (15\%) vs. } \\
\text { least frail }(60 \%) \text { group }\end{array}$} \\
\hline & \multirow[t]{2}{*}{ Institutionalization: 4550} & & & (EFS: HR= 2.49) & $(2.32-2.68)$ & \\
\hline & & & & (CHESS: HR = 2.32) & $(2.15-2.51)$ & \\
\hline Drubbel et al. [22] & $\begin{array}{l}\text { Mortality/ED visits/ } \\
\text { institutionalization/ } \\
\text { out-of-hours GP surgery } \\
\text { visits: } 508\end{array}$ & $\begin{array}{l}\text { Cox proportional } \\
\text { hazards regression }\end{array}$ & Age, gender, consultation gap & $\mathrm{HR}=1.166$ & $1.129-1.210$ & $\begin{array}{l}\text { Per deficit increase in } \\
\mathrm{Fl} \text { score }\end{array}$ \\
\hline \multirow[t]{3}{*}{ Fang et al. [32] } & Recurrent falls: 109 & Logistic regression & Age, gender, education & $\mathrm{OR}=1.54$ & $1.34-1.76$ & $\begin{array}{l}\text { Per one-unit increment in } \\
\text { Fl score }\end{array}$ \\
\hline & Recurrent fractures: 174 & Logistic regression & Age, gender, education & $\mathrm{OR}=1.07$ & $0.94-1.22$ & $\begin{array}{l}\text { Per one-unitincrement in } \\
\mathrm{Fl} \mathrm{score}\end{array}$ \\
\hline & Mortality: 1101 & $\begin{array}{l}\text { Cox proportional } \\
\text { hazards regression }\end{array}$ & $\begin{array}{l}\text { Age, gender, education, falls, } \\
\text { fractures }\end{array}$ & $\mathrm{HR}=1.29$ & $1.25-1.33$ & $\begin{array}{l}\text { Per one-unit increment } \\
\text { Fl score }\end{array}$ \\
\hline García-González et al. [33] & Mortality: 279 & $\begin{array}{l}\text { Cox proportional } \\
\text { hazards regression }\end{array}$ & Age, gender & $\mathrm{HR}=6.45$ & $4.10-10.14$ & $\begin{array}{l}\text { Most frail (FI 0.35-0.65) vs. } \\
\text { least frail group }(0.00-0.07)\end{array}$ \\
\hline \multirow[t]{4}{*}{ Gu et al. [23] } & \multirow[t]{4}{*}{ Mortality: 5,753 } & \multirow{4}{*}{$\begin{array}{l}\text { Weibull proportional } \\
\text { hazards regression }\end{array}$} & \multirow{4}{*}{$\begin{array}{l}\text { Age, ethnicity, urban-rural } \\
\text { residence, SES, family/social } \\
\text { connection and support, health } \\
\text { practices }\end{array}$} & Men (65-79): & & \multirow{4}{*}{$\begin{array}{l}\text { Most frail vs. least frail } \\
\text { quartile }\end{array}$} \\
\hline & & & & $\mathrm{HR}=4.56$ & 0.96 & \\
\hline & & & & Women (65-79): & & \\
\hline & & & & $\mathrm{HR}=3.84$ & 1.01 & \\
\hline \multirow[t]{3}{*}{ Hogan et al. [37] } & Mortality: 170 & Logistic regression & Age, gender, co-morbidity & $\mathrm{RR}=2.35$ & $1.56-3.54$ & \multirow{3}{*}{$\begin{array}{l}\text { All analyses: most frail } \\
(\mathrm{Fl}>0.30) \text { vs. least } \\
\text { frail group }(\mathrm{FI}<0.20)\end{array}$} \\
\hline & $\geq 1$ hospitalization: 424 & Logistic regression & Age, gender, co-morbidity & $\mathrm{RR}=1.28$ & $1.04-1.57$ & \\
\hline & Institutionalization: 204 & Logistic regression & Age, gender, co-morbidity & $\mathrm{RR}=3.30$ & $2.29-4.76$ & \\
\hline \multirow[t]{2}{*}{ Kulminski et al. [24] } & \multirow[t]{2}{*}{ Mortality: 421} & \multirow{2}{*}{$\begin{array}{l}\text { Cox proportional } \\
\text { hazards regression }\end{array}$} & \multirow[t]{2}{*}{ Age, gender, FP } & $\mathrm{Fl}: \mathrm{RR}=1.035$ & $1.026-1.045$ & \\
\hline & & & & $(F P: R R=1.014)$ & $(1.009-1.019)$ & $\begin{array}{l}\text { Per } 1 \% \text { increment in } \\
\text { Fl score (or FP) }\end{array}$ \\
\hline Kulminski et al. [25] & Mortality: 2146 & $\begin{array}{l}\text { Cox proportional } \\
\text { hazards regression }\end{array}$ & Age, gender & $R R=1.029$ & 1.001 & $\begin{array}{l}\text { Per } 1 \% \text { increment in } \\
\text { Fl score }\end{array}$ \\
\hline \multirow[t]{2}{*}{ Lucicesare et al. [26] } & \multirow[t]{2}{*}{ Mortality: 147} & \multirow{2}{*}{$\begin{array}{l}\text { Cox proportional } \\
\text { hazards regression }\end{array}$} & \multirow[t]{2}{*}{ Age, gender, CSBA score } & $\mathrm{Fl}: \mathrm{HR}=5.26$ & $1.05-26.42$ & \multirow[t]{2}{*}{$?$} \\
\hline & & & & (CSBA score: $\mathrm{HR}=1.52$ ) & $(1.28-1.81)$ & \\
\hline \multirow[t]{5}{*}{ Mitnitski et al. [34] } & \multirow[t]{5}{*}{ Mortality (\%/yr) 3.7-20.6 } & \multirow{5}{*}{$\begin{array}{l}\text { Cox proportional } \\
\text { hazards regression }\end{array}$} & \multirow[t]{5}{*}{ Age, gender } & CSHA-s: HR = 1.031 & 0.003 & \multirow{5}{*}{$\begin{array}{l}\text { Per deficit increase in } \\
\text { FI score }\end{array}$} \\
\hline & & & & CSHA-C: HR $=1.054$ & 0.007 & \\
\hline & & & & CSHA-i: HR= 1.046 & 0.009 & \\
\hline & & & & SOPSA: HR =1.079 & 0.022 & \\
\hline & & & & NHANES: HR = 1.011 & 0.003 & \\
\hline Searle et al. [19] & Mortality: ? & $\begin{array}{l}\text { Cox proportional } \\
\text { hazards regression }\end{array}$ & Age, gender & $\mathrm{HR}=1.03$ & $1.02-1.04$ & $\begin{array}{l}\text { Per } 0.01 \text { increase in } \\
\text { Fl score }\end{array}$ \\
\hline
\end{tabular}


Table 3 Criterion validity results; the predictive ability of the frailty index for adverse health outcomes (Continued)

\begin{tabular}{|c|c|c|c|c|c|c|}
\hline Shi et al. [35] & Mortality: 1,155 & Cox proportional hazards regression & Age, gender & $\mathrm{HR}=1.13$ & $1.09-1.47$ & $\begin{array}{l}\text { Per deficit increase in } \\
\text { Fl score }\end{array}$ \\
\hline Song et al. [29] & Mortality: 1,208 & Cox proportional hazards regression & Age, gender & $\mathrm{Fl}: \mathrm{RR}=1.57$ & $1.41-1.74$ & $\begin{array}{l}\text { Per Fl level }(F \mathrm{FI} \leq 0.08 \text {; } \\
\mathrm{FI} \text { between } 0.08-0.25 \\
\mathrm{FI} \geq 0.25)\end{array}$ \\
\hline Theou et al. [36] & Mortality: 1002 & Cox proportional hazards regression & $\begin{array}{l}\text { Age, gender, nr. of ADL } \\
\text { disabilities, nr. of chronic } \\
\text { diseases }\end{array}$ & $\mathrm{FI} 1: \mathrm{HR}=1.11$ & $1.06-1.17$ & $\begin{array}{l}\text { Per } 0.1 \text { increase in } \\
\text { Fl score }\end{array}$ \\
\hline \multirow[t]{5}{*}{ Woo et al. [31] } & $\begin{array}{l}\text { Change in ADL score } \\
0-3 \text { yrs }^{\mathrm{a}}\end{array}$ & Linear regression & $\begin{array}{l}\text { Age, gender, ADL score } \\
\text { at baseline }\end{array}$ & $B=-4.99$ & $-7.68--2.30$ & $\begin{array}{l}\text { Per } 1.0 \text { increase in } \\
\text { Fl score }\end{array}$ \\
\hline & $\begin{array}{l}\text { Change in mental score } \\
0-3 \mathrm{yrs}^{\mathrm{a}}\end{array}$ & Linear regression & $\begin{array}{l}\text { Age, gender, mental score } \\
\text { at baseline }\end{array}$ & $B=-2.23$ & $-4.11--0.35$ & $\begin{array}{l}\text { Per } 1.0 \text { increase in } \\
\text { Fl score }\end{array}$ \\
\hline & $\begin{array}{l}\text { Change in hospital } \\
\text { days } 0-3 \mathrm{yrs}^{\mathrm{a}}\end{array}$ & Linear regression & $\begin{array}{l}\text { Age, gender, hospital days } \\
\text { at baseline }\end{array}$ & $B=45.74$ & $28.16-63.33$ & $\begin{array}{l}\text { Per } 1.0 \text { increase in } \\
\text { Fl score }\end{array}$ \\
\hline & \multirow[t]{2}{*}{ New diseases at three $\mathrm{yrs}^{\mathrm{a}}$} & \multirow[t]{2}{*}{ Ordinal logistic regression } & \multirow[t]{2}{*}{-} & \multicolumn{2}{|c|}{$\begin{array}{l}\text { For } \mathrm{FI}=0.00 \text {, predicted } \\
\text { probability } \geq 1 \text { new }\end{array}$} & \multirow[t]{2}{*}{$\begin{array}{l}\text { Predicted probabilities } \\
\text { for new diseases } \\
\text { at } 3 \text { years }\end{array}$} \\
\hline & & & & \multicolumn{2}{|c|}{$\begin{array}{l}\text { For } \mathrm{FI}=0.50, \\
\text { predicted probability } \\
\geq 1 \text { new disease }=52.2 \%\end{array}$} & \\
\hline
\end{tabular}

Regression models with 3-year outcomes reported due to excess LTFU at 10 years. $95 \% \mathrm{Cl}=95 \%$ Confidence Interval; adm. = admission; ADL = Activities of Daily Living; B = beta; CHESS = Changes in Health,

End-Stage Disease and Signs and Symptoms Scale; CSBA = Conselice Study of Brain Ageing; CSHA = Canadian Study of Health and Ageing; DI = Deficit Index (Frailty Index); EFS = Edmonton Frail Scale; FI = Frailty Index; $\mathrm{FP}=$ Frailty Phenotype; HR = hazard ratio; NHANES = National Health and Nutrition Examination Survey; OR = odds ratio; PBA = Personal Biological Age; RR = relative risk; SE = standard error; SOPSA Sydney Older Persons Studies on Aging. 
score reported was 0.70 , indicating that no ceiling effect exists.

Our review has a number of strengths. First, we used a broad, sensitive search strategy with a low risk of missing relevant studies. Thus, we identified a large number of studies with consistent results across a variety of FIs in different populations. Second, we only considered relevant psychometric properties. We omitted reliability because the FI is an automated screening procedure and therefore not susceptible to intra- or interrater variability. Internal consistency was not examined because the FI is a formative model, i.e., the items form the construct together and therefore do not need to be correlated [39]. Third, the definitions used were tailored specifically to those aspects considered essential for frailty measures and based on a standardized taxonomy $[15,17]$. Fourth, we tailored our detailed inclusion and exclusion criteria to support our aim, which was to select those FI studies relevant for primary care. For example, we excluded studies with an FI based on a comprehensive geriatric assessment because it is not feasible to perform such an assessment for each older patient in primary care. Fifth, we appraised included studies critically using the QUIPS tool, which provided comprehensive quality assessment that demonstrated overall good quality of the methodology used in the included studies. The majority of studies reported sufficient details on their study sample, used appropriate criteria for FI construction, and reported few missing data. Moreover, the reported loss to follow-up was typically well below $20 \%$; thus, biased results were unlikely [40].

Our review also has several limitations. First, there is a risk of publication bias because studies with negative results are less likely to be published [41]. Because no register exists for validation studies, publication bias could not be formally assessed. Second, due to the withdrawal of one of the authors (GK), the first author (ID) performed the full-text assessment and quality appraisal partially alone, which may have caused potential selection bias. However, strict predefined selection and quality appraisal criteria were applied (see Additional files 1 and 2), and in cases where doubt existed, full-texts were assessed independently by the last author (MS). Third, most of the included studies on construct validity lacked prespecified hypotheses, which increases the risk of bias because, retrospectively, alternative explanations for low correlations may be sought [39]. Because the majority of correlations were robust, this risk appears limited. Finally, an individual patient data meta-analysis would have been preferable when summarizing research on the criterion validity of the FI. However, because the nature and number of deficits differed between the studies, it was not feasible to merge these data. Moreover, due to study heterogeneity, a meta-analysis on the outcome measures was not possible [41].
Apart from the FI, another frailty screening instrument that has shown good criterion and construct validity is the Frailty Phenotype [42]. One may question whether this performance-based measure would be preferable to implement in general practice, since it has also good face validity, consisting of five easily interpretable parameters (unintentional weight loss, self-reported exhaustion, weakness, slow walking speed, and low physical activity). However, compared to the FI, the Frailty Phenotype would require extra time and resources to enable execution in daily clinical care, and in direct comparison, the FI has been shown to better predict mortality risk among older people [24].

Our results are consistent with previous FI reviews that also reported on criterion validity and construct validity of the FI $[7,13,43]$. Our review updates these findings, and whereas these previous reviews were narrative in nature, our review is the first to systematically review the FI's psychometric properties that are relevant to primary care.

In the majority of the included studies on the FI's criterion validity, its predictive ability for mortality is examined. This does not mean that the FI is meant to be a 'mortality prediction' instrument; rather, by including the FI in a multivariable model including age, the FI score aims to explain the variable vulnerability to adverse health outcomes in people of the same age. This heterogeneity in frailty levels is also reflected by the relatively low correlation coefficients that we found between FI and age; whereas, in general, the correlation coefficient for the mean FI scores versus age was high (e.g. $r=0.985$, [34]), the correlation coefficient for the individual FI scores versus age was at maximum 0.320 [26].

To assess the construct validity of the FI, we focused on its correlation with other frailty measures, age, gender, disability, comorbidity, and self-rated health [15]. However, the concordance of the FI with a broad array of other measures has also been investigated, and a high FI score has been demonstrated to correlate with a high and low BMI [44], smoking [45,46], impaired psychological well-being [47], psychiatric illness [48], impaired mobility [49], impaired cognition and Alzheimer's disease [50,51], pain [52], high levels of gonadotropins [53], neighborhood deprivation and low individual socio-economic status [54], rural residence [55,56], and low education and little social support or participation [57]. The FI may also serve as a basis to calculate 'biological age'. Individuals with an FI score that is relatively high for their age and gender show a biological age that is higher than their chronological age, and this biological age is also a significant predictor of mortality [58].

There is no evidence supporting responsiveness or utility. However, some studies reflected upon the potential utility of the FI and noted two major advantages: 
first, the FI can be constructed from available data whether from administrative routine primary care data [22], specific measurements, such as the interRAI-AL instrument [37], or comprehensive geriatric assessment data $[26,29]$. Second, the FI score can be calculated using software thereby facilitating its clinical application $[24,37]$. However, only in one study the FI was actually studied in routine clinical data, so these potential advantages need to be further explored.

One may argue that studies relating FI score change to baseline factors, such as mobility and baseline frailty state, and studies modeling FI score change $[49,59]$ do describe responsiveness. These studies demonstrate that FI score development over time can be adequately described using a time dependent Poisson distribution, and that the probability of improvement, stability and worsening of the FI score is directly related to the baseline number of deficits, age, and mobility status. However, we did not consider these studies as responsiveness studies, since they did not study pre-specified hypotheses regarding the expected correlations between changes in the score on the FI instrument, and changes in other variables, such as scores on other instruments, or demographic or clinical variables [17]. An important finding of our systematic review is that eighteen out of twenty studies explored the FI's psychometric properties in datasets gathered specifically for research purposes. These studies consistently showed a higher maximum and mean FI score compared with the study that investigated the FI using routine primary care data [22]. however, because only one study with an FI using routine primary care data was included, there is not enough evidence to support conclusions about any structural differences in mathematical properties of the FI. More FIs applied in routine primary care data sets should be studied to further explore these potentially different mathematical properties. The narrower FI score range in the study using routine primary care data reflects unexpectedly low deficit prevalences, which may be caused by several reasons: first, patients may experience symptoms or problems with which they do not present themselves to the GP; second, there may be suboptimal data registration in the EMR $[60,61]$, and third, the FI may need to include more items on level of functioning, mobility or health attitude instead of merely relying on morbidity deficits. Also, except for the polypharmacy deficit, this FI was based on one single data source out of the Electronic Medical Records (EMRs), namely symptoms and diagnoses encoded according to the International Classification of Primary Care (ICPC, [62]). Care should be taken to construct an FI that captures all information available in the EMR by using, for example, not only ICPC-encoded data but also diagnostic measurement data, such as body mass index or laboratory tests, and elaborate medication data, encoded according to the Anatomic Therapeutic Chemical (ATC) [63].

\section{Conclusions}

In this systematic review, the FI demonstrates good criterion and construct validity, but its discriminatory ability is poor to moderate. In general, the FI appears to be an easily interpretable instrument that is practical to manage; however, studies that focus on its responsiveness, interpretability or utility are lacking. These results support the potential of the FI as a screening instrument for frailty in primary care and also demonstrate that further research into its psychometric properties is required. FIs based on research data show lower FI scores than those based on routine primary care data. Given its implementation in clinical practice, future validation studies of the FI should focus primarily on its application in routine primary care data.

\section{Additional files}

Additional file 1: Systematic review on the psychometric properties of the frailty index.

Additional file 2: QUIPS risk of bias assessment instrument for prognostic factor studies.

\section{Competing interests}

The authors declare that they have no competing interests.

\section{Authors' contributions}

ID, MJS, MEN and NJW contributed to the study concept and design. ID drafted the manuscript. ID and GK selected relevant studies independently, and ID extracted data and assessed the quality of the included studies. ID, NJW, NB, MEN and MJS provided a critical review of this manuscript. All authors read and approved the final version of this manuscript.

\section{Acknowledgements}

We thank Dr. Hans Reitsma for his advice on the possibilities of performing a meta-analysis, and we thank Dr. Saskia Weldam for her suggestions on the eligibility criteria and quality assessment.

\section{Author details}

${ }^{1}$ Department of General Practice, Julius Center for Health Sciences and Primary Care, University Medical Center Utrecht, Str. 6.131, Universiteitsweg 100, 3584 CG Utrecht, the Netherlands. '2Department of Public Health and Primary Care, Leiden University Medical Center, Hippocratespad 21, 2333 RC Leiden, the Netherlands. ${ }^{3}$ University Medical Center Utrecht, Department of Rehabilitation, Nursing Science and Sports Medicine, Heidelberglaan 100, 3584 CX Utrecht, the Netherlands.

Received: 2 July 2013 Accepted: 20 February 2014 Published: 6 March 2014

\section{References}

1. De Lepeleire J, Degryse J, Illiffe S, Mann E, Buntinx F: Family physicians need easy instruments for frailty. Age Ageing 2008, 37:484-485.

2. Moorhouse $P$, Rockwood K: Frailty and its quantitative clinical evaluation. J R Coll Surg Edinb 2012, 42:333-340.

3. Lacas A, Rockwood K: Frailty in primary care: a review of its conceptualization and implications for practice. BMC Med 2012, 10:4.

4. Loo TS, Davis RB, Lipsitz LA, Irish J, Bates CK, Agarwal K, Markson L, Hamel $M B$ : Electronic medical record reminders and panel management to improve primary care of elderly patients. Arch Intern Med 2011, 171:1552-1558.

5. Heuberger RA: The frailty syndrome: a comprehensive review. J Nutr Gerontol Geriatr 2011, 30:315-368. 
6. Mitnitski AB, Mogilner AJ, Rockwood K: Accumulation of deficits as a proxy measure of aging. Scientific World J 2001, 8:323-36.

7. Rockwood K, Mitnitski A: Frailty in relation to the accumulation of deficits. J Gerontol A Biol Sci Med Sci 2007, 62:722-727.

8. Hoogendijk EO, van der Horst HE, Deeg DJ, Frijters DH, Prins BA, Jansen AP, Nijpels G, van Hout HP: The identification of frail older adults in primary care: Comparing the accuracy of five simple instruments. Age Ageing 2013, 42:262-265.

9. Pialoux T, Goyard J, Lesourd B: Screening tools for frailty in primary health care: A systematic review. Geriatr Gerontol Int 2012, 12:189-197.

10. Pijpers E, Ferreira I, Stehouwer CD, Nieuwenhuijzen Kruseman AC: The frailty dilemma. review of the predictive accuracy of major frailty scores. Eur J Int Med 2012, 23:118-123.

11. Sternberg SA, Wershof Schwartz A, Karunananthan S, Bergman H, Mark Clarfield A: The identification of frailty: a systematic literature review. J Am Geriatr Soc 2011, 59:2129-2138.

12. Clegg A, Young J, lliffe S, Rikkert MO, Rockwood K: Frailty in elderly people. Lancet 2013, 381:752-762.

13. Rockwood K, Mitnitski A: Frailty defined by deficit accumulation and geriatric medicine defined by frailty. Clin Geriatr Med 2011, 27:17-26.

14. De Vries NM, Staal JB, van Ravensberg CD, Hobbelen JS, Olde Rikkert MG, Nijhuis-van der Sanden MW: Outcome instruments to measure frailty: a systematic review. Ageing Res Rev 2011, 10:104-114.

15. Rockwood K: What would make a definition of frailty successful? Age Ageing 2005, 34:432-434

16. McDowell I, Newell C: Measuring health: A guide to rating scales and questionnaires. 2nd edition. New York: Oxford University Press; 1996.

17. Terwee $C B$, Bot SD, de Boer MR, van der Windt DA, Knol DL, Dekker J, Bouter LM, de Vet HC: Quality criteria were proposed for measurement properties of health status questionnaires. J Clin Epidemiol 2007, 60:34-42.

18. Hayden JA, van der Windt DA, Cartwright JL, Cote P, Bombardier C: Assessing bias in studies of prognostic factors. Ann Intern Med 2013, 158:280-286.

19. Searle SD, Mitnitski A, Gahbauer EA, Gill TM, Rockwood K: A standard procedure for creating a frailty index. BMC Geriatr 2008, 8:24.

20. Cigolle CT, Ofstedal MB, Tian Z, Blaum CS: Comparing models of frailty: The health and retirement study. J Am Geriatr Soc 2009, 57:830-839.

21. Armstrong JJ, Stolee P, Hirdes JP, Poss JW: Examining three frailty conceptualizations in their ability to predict negative outcomes for home-care clients. Age Ageing 2010, 39:755-758

22. Drubbel I, de Wit NJ, Bleijenberg N, Eijkemans RJ, Schuurmans MJ, Numans ME: Prediction of adverse health outcomes in older people using a frailty index based on routine primary care data. J Gerontol A Biol Sci Med SCi 2013, 68:301-308.

23. Gu D, Dupre ME, Sautter J, Zhu H, Liu Y, Yi Z: Frailty and mortality among Chinese at advanced ages. J Gerontol B Psychol Sci Soc Sci 2009, 64:279-289.

24. Kulminski AM, Ukraintseva SV, Kulminskaya IV, Arbeev KG, Land K, Yashin Al: Cumulative deficits better characterize susceptibility to death in elderly people than phenotypic frailty: lessons from the cardiovascular health study. J Am Geriatr Soc 2008, 56:898-903.

25. Kulminski A, Yashin A, Arbeev K, Akushevich I, Ukraintseva S, Land K, Manton K: Cumulative index of health disorders as an indicator of aging-associated processes in the elderly: results from analyses of the national long term care survey. Mech Ageing Dev 2007, 128:250-258.

26. Lucicesare A, Hubbard RE, Fallah N, Forti P, Searle SD, Mitnitski A, Ravaglia G, Rockwood K: Comparison of two frailty measures in the conselice study of brain ageing. J Nutr Health Aging 2010, 14:278-281.

27. Lucicesare A, Hubbard RE, Searle SD, Rockwood K: An index of self-rated health deficits in relation to frailty and adverse outcomes in older adults. Aging Clin Exp Res 2010, 22:255-260.

28. Rockwood K, Andrew M, Mitnitski A: A comparison of two approaches to measuring frailty in elderly people. J Gerontol A Biol Sci Med Sci 2007, 62:738-743

29. Song X, Mitnitski A, Rockwood K: Prevalence and 10-year outcomes of frailty in older adults in relation to deficit accumulation. J Am Geriatr SoC 2010, 58:681-687.

30. Woo J, Leung J, Morley JE: Comparison of frailty indicators based on clinical phenotype and the multiple deficit approach in predicting mortality and physical limitation. J Am Geriatr Soc 2012, 60:1478-1486.

31. Woo J, Goggins W, Sham A, Ho SC: Public health significance of the frailty index. Disabil Rehabil 2006, 28:515-521.
32. Fang X, Shi J, Song X, Mitnitski A, Tang Z, Wang C, Yu P, Rockwood K: Frailty in relation to the risk of falls, fractures, and mortality in older chinese adults: results from the Beijing longitudinal study of aging. J Nutr Health Aging 2012, 33:1-5.

33. Garcia-Gonzalez JJ, Garcia-Pena C, Franco-Marina F, Gutierrez-Robledo LM: A frailty index to predict the mortality risk in a population of senior Mexican adults. BMC Geriatr 2009, 9:47.

34. Mitnitski A, Song X, Skoog I, Broe GA, Cox JL, Grunfeld E, Rockwood K: Relative fitness and frailty of elderly men and women in developed countries and their relationship with mortality. J Am Geriatr Soc 2005, 53:2184-2189.

35. Shi J, Song X, Yu P, Tang Z, Mitnitski A, Fang X, Rockwood K: Analysis of frailty and survival from late middle age in the Beijing longitudinal study of aging. BMC Geriatr 2011, 11:17.

36. Theou O, Rockwood MR, Mitnitski A, Rockwood K: Disability and co-morbidity in relation to frailty: How much do they overlap? Arch Gerontol Geriatr 2012, 55:e1-e8.

37. Hogan DB, Freiheit EA, Strain LA, Patten SB, Schmaltz HN, Rolfson D, Maxwell CJ: Comparing frailty measures in their ability to predict adverse outcome among older residents of assisted living. BMC Geriatr 2012, 12:56.

38. Hirdes JP, Frijters DH, Teare GF, The MDS-CHESS scale: A new measure to predict mortality in institutionalized older people. J Am Geriatr Soc 2003, 51:96-100.

39. Mokkink LB, Terwee CB, Knol DL, Stratford PW, Alonso J, Patrick DL, Bouter LM, de Vet HC: The COSMIN checklist for evaluating the methodological quality of studies on measurement properties: a clarification of its content. BMC Med Res Methodol 2010, 10:22. 2288-10-22.

40. Laupacis A, Wells G, Richardson WS, Tugwell P: Users' guides to the medical literature. V. how to use an article about prognosis. evidencebased medicine working group. JAMA 1994, 272:234-237.

41. Egger M, Schneider M, Davey Smith G: Spurious precision? Meta-analysis of observational studies. BMJ 1998, 316:140-144.

42. Fried LP, Tangen CM, Walston J, Newman AB, Hirsch C, Gottdiener J, Seeman T, Tracy R, Kop WJ, Burke G, McBurnie MA, Cardiovascular Health Study Collaborative Research Group: Frailty in older adults: evidence for a phenotype. J Gerontol A Biol Sci Med Sci 2001, 56:M146-M156.

43. Rockwood K, Mitnitski A: Frailty, fitness, and the mathematics of deficit accumulation. Rev Clin Gerontol 2007, 17:1-12.

44. Hubbard RE, Lang IA, Llewellyn DJ, Rockwood K: Frailty, body mass index, and abdominal obesity in older people. J Gerontol A Biol Sci Med Sci 2010, 65:377-381

45. Hubbard RE, Searle SD, Mitnitski A, Rockwood K: Effect of smoking on the accumulation of deficits, frailty and survival in older adults: a secondary analysis from the Canadian study of health and aging. J Nutr Health Aging 2009, 13:468-472.

46. Wang C, Song X, Mitnitski A, Yu P, Fang X, Tang Z, Shi J, Rockwood K: Gender differences in the relationship between smoking and frailty: Results from the Beijing longitudinal study of aging. J Gerontol A Biol SCi Med Sci 2013, 68:338-346.

47. Andrew MK, Fisk JD, Rockwood K: Psychological well-being in relation to frailty: A frailty identity crisis? Int Psychogeriatr 2012, 24:1347-1353.

48. Andrew MK, Rockwood K: Psychiatric illness in relation to frailty in community-dwelling elderly people without dementia: a report from the canadian study of health and aging. Can J Aging 2007, 26:33-38.

49. Fallah N, Mitnitski A, Searle SD, Gahbauer EA, Gill TM, Rockwood K: Transitions in frailty status in older adults in relation to mobility: a multistate modeling approach employing a deficit count. J Am Geriatr Soc 2011, 59:524-529.

50. Mitnitski $A B$, Fallah N, Rockwood K: Changes in cognition in relation to frailty in older canadians. Alzheimers Dement 2009, 5:391.

51. Song X, Mitnitski A, Rockwood K: Frailty and alzheimer's disease in older men and women: results from a population-based study. Alzheimers Dement 2009, 5:129.

52. Shega JW, Dale W, Andrew M, Paice J, Rockwood K, Weiner DK: Persistent pain and frailty: a case for homeostenosis. J Am Geriatr Soc 2012, 60:113-117.

53. Tajar A, O'Connell MD, Mitnitski AB, O'Neill TW, Searle SD, Huhtaniemi IT, Finn JD, Bartfai G, Boonen S, Casanueva FF, Forti G, Giwercman A, Han TS, Kula K, Labrie F, Lean ME, Pendleton N, Punab M, Silman AJ, Vanderschueren D, Rockwood K, Wu FC, European Male Aging Study Group: 
Frailty in relation to variations in hormone levels of the hypothalamicpituitary-testicular axis in older men: results from the European male aging study. J Am Geriatr Soc 2011, 59:814-821.

54. Lang IA, Hubbard RE, Andrew MK, Llewellyn DJ, Melzer D, Rockwood K: Neighborhood deprivation, individual socioeconomic status, and frailty in older adults. J Am Geriatr Soc 2009, 57:1776-1780.

55. Song X, MacKnight C, Latta R, Mitnitski AB, Rockwood K: Frailty and survival of rural and urban seniors: results from the Canadian study of health and aging. Aging Clin Exp Res 2007, 19:145-153.

56. Yu P, Song X, Shi J, Mitnitski A, Tang Z, Fang X, Rockwood K: Frailty and survival of older Chinese adults in urban and rural areas: results from the Beijing longitudinal study of aging. Arch Gerontol Geriatr 2012, 54:3-8.

57. Woo J, Goggins W, Sham A, Ho SC: Social determinants of frailty. Gerontology 2005, 51:402-408.

58. Goggins WB, Woo J, Sham A, Ho SC: Frailty index as a measure of biological age in a Chinese population. $J$ Gerontol A Biol Sci Med Sci 2005, 60:1046-1051.

59. Mitnitski A, Bao L, Rockwood K: Going from bad to worse: a stochastic model of transitions in deficit accumulation, in relation to mortality. Mech Ageing Dev 2006, 127:490-493.

60. Kahn NA, Visscher S, Verheij RA: The quality of the electronic medical records of general practitioners. (report in Dutch - de kwaliteit van het elektronisch patiëntendossier van huisartsen gemeten). NIVEL: Utrecht; 2011.

61. Soler JK, Pringle M: Research using electronic patient records in general practice. The EGPRN meeting in Bertinoro, Italy, May 2009. Eur $J$ Gen Pract 2010, 16:186-189.

62. Lamberts HWM: ICPC international classification of primary care. Oxford: Oxford University Press; 1987.

63. WHO Collaborating Centre for Drug Statistics Methodology: Guidelines for ATC classification and DDD assignment 2011. Oslo; 2010.

doi:10.1186/1471-2318-14-27

Cite this article as: Drubbel et al: Screening for frailty in primary care: a systematic review of the psychometric properties of the frailty index in community-dwelling older people. BMC Geriatrics 2014 14:27.

\section{Submit your next manuscript to BioMed Central and take full advantage of:}

- Convenient online submission

- Thorough peer review

- No space constraints or color figure charges

- Immediate publication on acceptance

- Inclusion in PubMed, CAS, Scopus and Google Scholar

- Research which is freely available for redistribution 